\title{
Identification and Quantitative Assessment of Different Components of Intracranial Atherosclerotic Plaque by Ex Vivo 3T High-Resolution Multicontrast MRI
}

\author{
(D)Y. Jiang, (1DW. Peng, (D) B. Tian, (D) C. Zhu, (D) Chen, DX. Wang, (D). Liu, (D)Y. Wang, (D) Z. Xiang, (D) A.J. Degnan, (D) Z. Teng, \\ (DD. Saloner, and (D). Lu
}

수일

\begin{abstract}
BACKGROUND AND PURPOSE: High-resolution 3T MR imaging can visualize intracranial atherosclerotic plaque. However, histologic validation is still lacking. This study aimed to evaluate the ability of $3 T$ MR imaging to identify and quantitatively assess intracranial atherosclerotic plaque components ex vivo with histologic validation.
\end{abstract}

MATERIALS AND METHODS: Fifty-three intracranial arterial specimens with atherosclerotic plaques from 20 cadavers were imaged by $3 T$ MR imaging with T1, T2, and proton-density-weighted FSE and STIR sequences. The signal characteristics and areas of fibrous cap, lipid core, calcification, fibrous tissue, and healthy vessel wall were recorded on MR images and compared with histology. Fibrous cap thickness and maximum wall thickness were also quantified. The percentage of areas of the main plaque components, the ratio of fibrous cap thickness to maximum wall thickness, and plaque burden were calculated and compared.

RESULTS: The signal intensity of the lipid core was significantly lower than that of the fibrous cap on T2-weighted, proton-density, and STIR sequences $(P<.01)$ and was comparable on T1-weighted sequences $(P=1.00)$. Optimal contrast between the lipid core and fibrous cap was found on T2-weighted images. Plaque component mean percentages were comparable between MR imaging and histology: fibrous component $(81.86 \% \pm 10.59 \%$ versus $81.87 \% \pm 11.59 \%, P=.999)$, lipid core $(19.51 \% \pm 10.76 \%$ versus $19.86 \% \pm 11.56 \%, P=.863)$, and fibrous cap $(31.10 \% \pm 11.28 \%$ versus $30.83 \% \pm 8.51 \%, P=.463)$. However, MR imaging overestimated mean calcification $(9.68 \% \pm 5.21 \%$ versus $8.83 \% \pm 5.67 \%, P=.030)$ and plaque burden $(65.18 \% \pm 9.01 \%$ versus $52.71 \% \pm 14.58 \%, P<.001)$.

CONCLUSIONS: Ex vivo 3T MR imaging can accurately identify and quantitatively assess intracranial atherosclerotic plaque components, providing a direct reference for in vivo intracranial plaque imaging.

ABBREVIATIONS: ICC $=$ intraclass correlation coefficient; $\mathrm{PD}=$ proton-density

ntracranial atherosclerotic disease is one of the most common causes of stroke. ${ }^{1}$ It has a high prevalence in the Asian popula-

Received March 10, 2016; accepted after revision April 23, 2017.

From the Department of Radiology (Y.J.), Wuhan General Hospital of the People's Liberation Army, Wuhan, China; Departments of Radiology (Y.J., W.P., B.T., L.C.,

X.W., Q.L., J.L.) and Pathology (Y.W., Z.X.), Changhai Hospital, Shanghai, China; Department of Radiology and Biomedical Imaging (C.Z., D.S.), University of California, San Francisco, San Francisco, California; Department of Radiology (A.J.D.), University of Pittsburgh Medical Center, Pittsburgh, Pennsylvania; and Department of Radiology (Z.T.), University of Cambridge, Cambridge, UK.

Y. Jiang, W. Peng, and B. Tian contributed equally to this work.

This work was supported by the Twelfth Five-Year Plan Medical Key Project of the People's Liberation Army, China (BWS12J026); Shanghai Hospital Development Center grant (SHDC12013110); the National Nature Science Foundation of China (NSFC31470910, NSFC81270413); and the National Institutes of Health grants R01HL114118 and R01NS059944.

Please address correspondence to Jianping Lu, MD, Department of Radiology, Changhai Hospital of Shanghai, 168 Changhai Rd, Shanghai, China, 20043; e-mail: cjr.lujianping@vip.163.com; and Chengcheng Zhu, PhD, Department of Radiology and Biomedical Imaging, University of California, San Francisco, Room BA34, VA Medical Center, 4150 Clement St, San Francisco, CA 94121; e-mail: Chengcheng.Zhu@ucsf.edu tion $^{2}$ with a high recurrence rate. ${ }^{3}$ Due to its high morbidity and mortality, early diagnosis and prognostic evaluation are particularly important. High-resolution vessel wall MR imaging is a promising noninvasive method to evaluate intracranial plaque in vivo, with excellent soft-tissue contrast. ${ }^{4}$ In recent years, a few studies have attempted to characterize intracranial atherosclerotic plaque composition by in vivo MR imaging. ${ }^{5-9}$ Intracranial plaque imaging with MR imaging has identified high-risk plaque correlated with infarct territory and plaque associated with stroke recurrence. ${ }^{10-13}$ However, these studies have mostly focused on plaque enhancement to ascertain plaque risk. Moreover, the determination of plaque composition in other studies has been based on the MR imaging characteristics of extracranial carotid plaque, while direct histologic validation of in vivo MR imaging of

\footnotetext{
-- Indicates open access to non-subscribers at www.ajnr.org

三 Indicates article with supplemental on-line tables.

Indicates article with supplemental on-line photos.

http://dx.doi.org/10.3174/ajnr.A5266
} 
intracranial plaque is still lacking, except for 1 individual case study. ${ }^{14}$

Ex vivo MR imaging of intracranial atherosclerotic plaque specimens provides a way to understand intracranial plaque. A few studies have reported ex vivo MR imaging characteristics of intracranial plaque with $7 \mathrm{~T}$ research scanners. ${ }^{15-17}$ However, these results cannot be transferred to $3 \mathrm{~T}$ for the clinical evaluation of intracranial arterial disease because the MR relaxometry is different at $3 \mathrm{~T}$ and $7 \mathrm{~T}$. Very few studies have reported intracranial plaque characteristics at 3T, the highest field strength that is clinically relevant. ${ }^{14,18}$ Thus, there is an urgent need to investigate whether clinical $3 \mathrm{~T}$ scanners provide sufficient contrast for the evaluation of intracranial plaque composition. In addition, most of these 7T studies focused on the qualitative classification of plaque components and lacked quantitative assessment of highrisk plaque features, including the lipid core area, fibrous cap thickness, and plaque burden, all of which play an important role in atherosclerotic plaque progression and rupture. ${ }^{19,20}$

The purpose of this study was to report the signal characteristics of plaque components on multicontrast MR imaging at $3 \mathrm{~T}$ and evaluate the ability of MR imaging to quantify plaque components with histologic validation.

\section{MATERIALS AND METHODS \\ Study Population}

The study was approved by our hospital ethics committee, and informed consent was obtained from the patients' relatives. $\mathrm{Pa}$ tients 45 years of age or older who underwent a postmortem examination in our hospital (Changhai Hospital) from January 2013 to June 2015 were recruited for the study. Among 120 cadavers, 20 with possible atherosclerotic specimens of the circle of Willis were selected on the basis of macroscopic examination by a pathologist during the postmortem examination. The time between death and the postmortem examination was $<1$ month; however, the exact time window was unknown due to the limitations of our local ethics committee. The same specimens were evaluated in a previous study ${ }^{18}$; however, the current study addresses distinctly different research questions, specifically performing a quantitative analysis of the identification of plaque components on multicontrast imaging. The demographics of the cadavers were reported in the previous publication. ${ }^{18}$ All specimens were rinsed carefully with saline to remove blood clots. The same pathologist excised possible atherosclerotic arteries according to gross morphologic changes. All excised arteries were then stored at $4^{\circ} \mathrm{C}$. The time between the postmortem examination and imaging was $<24$ hours. Photographs of gross specimens of the circle of Willis are shown in On-line Fig 1.

\section{MR Imaging Protocol}

MR imaging was undertaken in a 3T whole-body system (Magnetom Skyra; Siemens, Erlangen, Germany) with a loop coil ( $4 \mathrm{~cm}$ in diameter). Before imaging, arteries were embedded in Fomblin (Solvey Solexis, Milan, Italy), which is a fluorinated fluid with no MR signal. Careful flushing of the lumen and outer surfaces of specimens was performed to ensure that gas bubbles were removed. The experimental setup is shown in On-line Fig 2. Multiple imaging sequences, including $\mathrm{T} 1, \mathrm{~T} 2$, and proton-density
(PD) weighted FSE and STIR were acquired perpendicular to the longitudinal axis of the arterial segments. The scanning parameters were identical to those in a previous study. ${ }^{18}$

\section{Histology}

The specimens were fixed for 24 hours in formal saline $(10 \%$ formalin in $0.9 \%$ sodium chloride) following imaging. After decalcification, samples were cut into sections between 4 and $5 \mathrm{~mm}$ in length and numbered to assist the coregistration. Each section was then sliced at a $5-\mu \mathrm{m}$ thickness with an interval of $0.5 \mathrm{~mm}$. Specimens were stained with hematoxylin-eosin and Masson trichrome to visualize arterial architecture, including atherosclerotic components. The histology sections were digitized with a digital microscope at $20 \times$ magnification (BX53 microscope; Olympus, Tokyo, Japan).

Five experienced radiologists were involved in the image analysis: coregistration of MR imaging and histology (Y.J.), measurement of signal intensities (B.T.), classification of plaque components (W.P.), and measurement and quantification of plaque component area/thickness (Q.L. and X.W.).

\section{Image Coregistration}

An experienced radiologist (Y.J.) matched the MR imaging sections and histologic sections carefully according to location. The gross morphologic features, such as vessel size and shape, plaque configuration, and specific plaque components (eg, calcification), were considered during the coregistration.

\section{Image Analysis}

Image analysis was performed in 2 steps. First, the signal intensity of plaque components on MR images was quantified with histology as a reference for plaque component categorization. Second, images containing advanced lesion types were selected for plaque component area quantification.

\section{Analysis of Signal Intensities of Plaque Components}

One experienced pathologist identified and segmented the main components of the plaque in every matched histologic section, including intraplaque hemorrhage, fibrous cap, lipid core, calcification, fibrous tissue (ie, smooth-muscle cell and dense collagen not including fibrous cap or healthy vessel wall), and healthy vessel wall according to the American Heart Association classification, ${ }^{21}$ as well as judging the lesion types-types I-II: initial lesion with foam cells or fatty streaks with multiple foam cell layers; type III: atheroma with extracellular lipid pools; types IV-V: plaque with a fibrous cap and lipid core; type VI: complex plaque with a surface defect, hemorrhage, or thrombus; type VII: calcified plaque; and type VIII: fibrotic plaque without a lipid core. ${ }^{22}$ According to the histologic results, a radiologist (B.T.) segmented plaque components on corresponding T2-weighted images with CMRtools (Cardiovascular Imaging Solutions, London, UK), and the contours on T2-weighted images were copied to the same anatomic sections of other MR images. This process allowed quantification of the signal intensity of plaque components. Because only a single segment of the vessel was imaged in our study, the signal of the healthy vessel wall that did not include any atheroma (mostly media) was used as a reference. A second radiolo- 
Table 1: The criteria for plaque component identification by multicontrast MRI

\begin{tabular}{llll}
\hline & \multicolumn{1}{c}{ Lipid Core } & \multicolumn{1}{c}{ Fibrous Cap } & Calcification \\
\hline T1 & Isointense/hyperintense & Isointense & Hypointense \\
T2 & Isointense/hypointense & Isointense/hyperintense & Hypointense \\
PD & Isointense/hypointense & Isointense/hyperintense & Hypointense \\
STIR & Hypointense & Hyperintense & Hypointense \\
\hline
\end{tabular}

histology were included in the analysis. The details of specimens and plaque types were reported in the previous publication. ${ }^{18}$

\section{Signal Intensity Characteristics}

Representative multicontrast MR im- gist (W.P.), blinded to histology results, identified the main plaque components on the basis of a criterion developed in prior histology-MR imaging comparative analysis (Table 1).

\section{Quantification of Plaque Component Area}

Sections containing advanced lesion types (types IV-VII) were included in the area analysis. Histologic sections were segmented and measured with ImageJ software (Version 1.47; National Institutes of Health, Bethesda, Maryland) by a pathologist blinded to MR imaging results. The lumen, vessel wall, and each plaque component were contoured, and the areas were recorded. We calculated the following metrics: vessel wall area (Vessel Wall Area $=$ Total Vessel Area - Lumen Area); fibrous component area (ie, smooth-muscle cells and dense collagen including the fibrous cap and healthy wall) (Fibrous Component Area = Vessel Wall Area - Other Component Area); the percentage of the area of each major plaque component (\% Plaque Component $=$ Specific Component Area / Vessel Wall Area $\times 100 \%$ ); and plaque burden (Plaque Burden $=$ Vessel Wall Area $/$ Total Vessel Area $\times$ $100 \%) .{ }^{23}$ Both the fibrous cap thickness and the maximum wall thickness were also measured on sections containing a lipid core. Because of shrinkage during histologic processing, ${ }^{24}$ the percentage of the plaque component area/thickness in addition to absolute area/thickness was used for histology-imaging comparison. Two radiologists (Q.L. and X.W.) segmented the components on MR imaging and measured the area/thickness blinded to histology results. One of the radiologists (X.W.) repeated the measurements 1 month later.

\section{Statistics}

Statistical analysis was performed in SPSS (Version 21.0; IBM, Armonk, New York). Normality assumptions were assessed by using a Shapiro-Wilk test. Categoric data were presented as counts, and continuous variables were presented as mean $\pm \mathrm{SD}$ or median (interquartile range). Considering that multiple measurements were obtained from each subject, a linear mixed-effects model was used to assess the differences among the signal intensities of different components and the differences between histology measurements and MR imaging measurements. The Cohen $\kappa$ with 95\% CIs was used to estimate the agreement of plaque component identification on MR imaging compared with histology. To evaluate the agreement of measurements between MR imaging and histology and intraobserver/interobserver reproducibility of MR imaging measurements, we calculated the intraclass correlation coefficient (ICC) with 95\% CIs. A $P$ value $<.05$ was considered significant. All $P$ values were 2 -sided.

\section{RESULTS}

Fifty-three intracranial arterial sections (1 7 arterial sections from each specimen) from 20 subjects with atherosclerotic plaques were excised; 207 sections with matched MR imaging and ages of the specimens and their corresponding histologic sections are shown in Figs 1 and 2. MR imaging characteristics of the main plaque components are shown in Table 1. Signal intensities of plaque components on multiple MR images are summarized in On-line Table 1. Overall, there were significant differences among signal intensities of different plaque components on multiple sequences $(P<.05)$. The best contrast between lipid core and fibrous cap assessment was achieved with T2-weighted images. Post hoc pair-wise tests showed that the signal intensity of the lipid core was significantly lower than that of the fibrous cap on T2-weighted, PD, and STIR sequences $(P<.01)$ and was not significantly different on T1-weighted imaging $(P=1.00)$. The signal intensities of the fibrous cap, fibrous tissue, and healthy wall were similar on all sequences $(P>.05)$. Lipid core and healthy wall had similar signal intensities on all sequences. Calcification showed the lowest signal intensity on T2-weighted, PD, and T1-weighted sequences $(P<.001)$. Pair-wise test results of the signal intensity of each 2 components are provided in On-line Table 2.

\section{Utility of MR Imaging for Plaque Component Characterization}

The sensitivity and specificity were $90.53 \%$ and $69.64 \%$ for identifying fibrous cap and lipid core and $83.33 \%$ and $100 \%$ for calcification on a combination of weightings. Cohen $\kappa$ values were 0.59 for the fibrous cap and lipid core and 0.90 for calcification.

\section{Quantitative Analysis}

Only sections containing $\geq 2$ components (types IV-V or VII) were selected for quantitative analysis. Fifty-seven sections were used for further analysis (Figs 1 and 2). The agreement between manual measurements performed on MR images and histology sections is shown in On-line Table 3. The absolute values were larger on MR imaging compared with histology, likely due to the shrinkage of the specimen during the histologic processing. Good-to-excellent correlation was observed ( $r=0.72-0.93$, ICC $=0.69-0.85)$, but fibrous cap thickness measurements had only a moderate correlation $(r=0.50$, ICC $=0.46)$. Table 2 shows the comparison between MR imaging and histology for the percentages of areas and thicknesses. Generally, there was good-toexcellent agreement $(r=0.86-0.93$, ICC $=0.77-0.93)$, except that the agreement of the percentage of the fibrous cap was moderate ( $r=0.44$, ICC $=0.42$ ). The percentages of plaque component areas and thicknesses were comparable between MR imaging and histology, but MR imaging significantly overestimated calcification $(9.68 \% \pm 5.21 \%$ versus $8.83 \% \pm 5.67 \%, P=.030)$ and plaque burden $(65.18 \% \pm 9.01 \%$ versus $52.71 \% \pm 14.58 \%, P<$ $.001)$. 

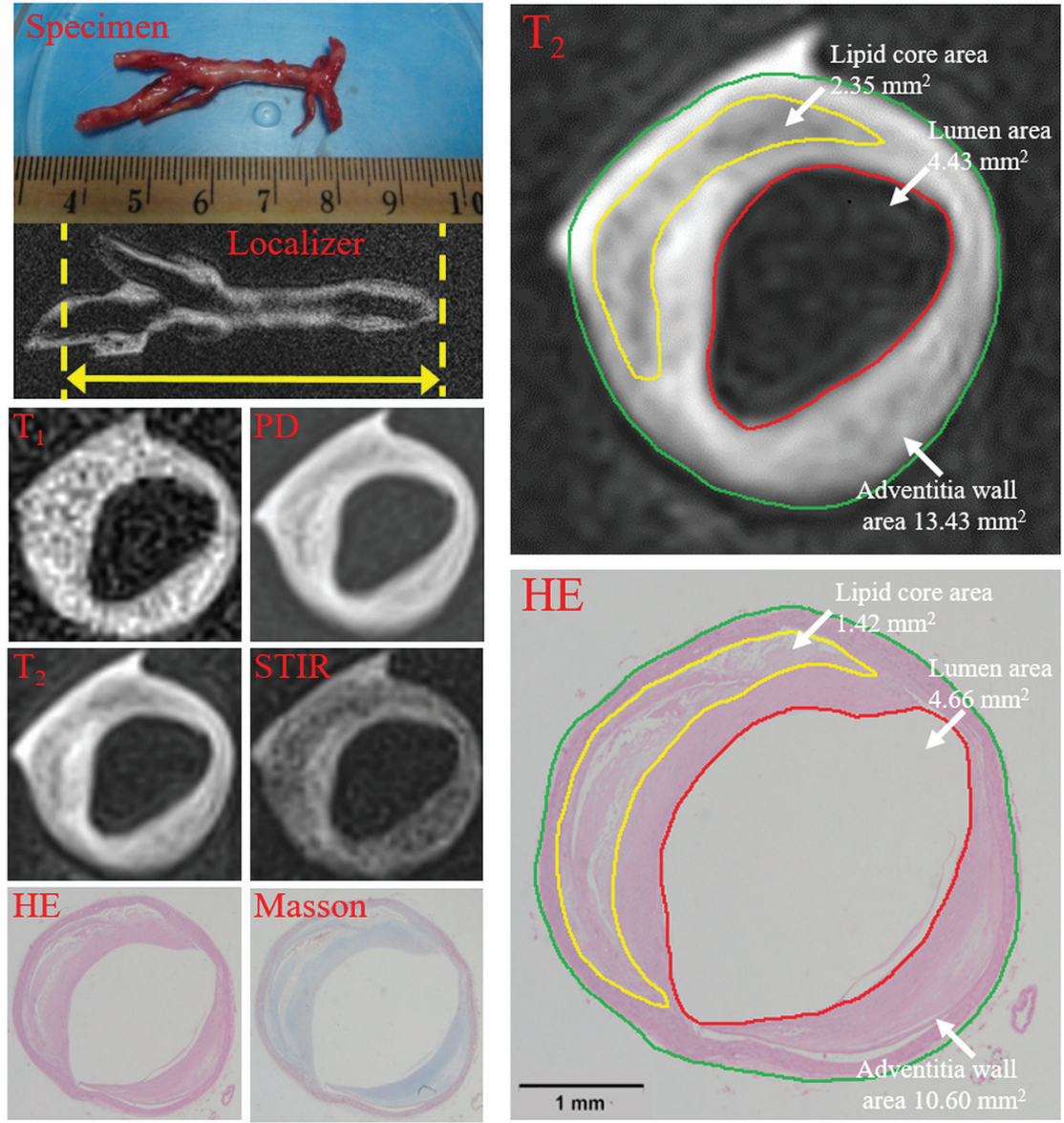

FIG 1. A basilar artery plaque with a thick fibrous cap and a large lipid core (type IV-V) was identified on multicontrast MR imaging and matched histology (20X magnification). Contours have been drawn for the lumen (red), vessel wall (green), and lipid core (yellow) on a T2-weighted image and hematoxylin-eosin-stained section. The lumen, total vessel, and lipid core areas were 4.34, 13.43, and $2.35 \mathrm{~mm}^{2}$ and 4.66, 10.60, and $1.42 \mathrm{~mm}^{2}$ for MR imaging and histology. The vessel wall areas calculated by total vessel area minus lumen area were 9.09 and $6.00 \mathrm{~mm}^{2}$. The fibrous component areas calculated by vessel wall area minus lipid core area were 6.70 and $4.56 \mathrm{~mm}^{2}$. The percentages of plaque components (Specific Component Area / Vessel Wall Area $\times 100 \%$ ) were $25.85 \%$ versus $23.63 \%$ for the lipid core and $74.15 \%$ versus $76.37 \%$ for fibrous tissue. Plaque burden (Vessel Wall Area / Total Vessel Area $\times 100 \%$ ) was $67.68 \%$ versus $56.24 \%$.

\section{Reproducibility of Quantitative MR Imaging Measurements}

Both intraobserver and interobserver reproducibility were good to excellent for the percentage of plaque component areas and thicknesses as well as plaque burden (ICC $=0.779-0.985$ ). Detailed measurements and analysis are shown in On-line Tables 4 and 5 .

\section{DISCUSSION}

To our knowledge, this is the first study to assess intracranial atherosclerotic plaque characteristics quantitatively with 3T MR imaging with histologic validation. Our results confirm that $3 \mathrm{~T}$ high-resolution multicontrast MR imaging can provide sufficient contrast to discriminate and quantitatively assess intracranial atherosclerotic plaque components ex vivo; this discrimination provides a reference for in vivo intracranial plaque imaging.

The ability of multicontrast MR imaging to ascertain the composition of extracranial carotid plaque has been extensively studied and validated against histology, with several of these plaque features correlated to increased risk of stroke. ${ }^{25}$ Histologic valida- tion of intracranial plaque imaging, however, is still very limited, mostly due to the challenge of collecting postmortem specimens. Only a few histology validation studies have been undertaken at 7T. ${ }^{15-17}$ The scan parameters of multicontrast MR imaging in this study were close to those in standard protocols used in clinical in vivo studies at $3 \mathrm{~T}$, except that this study used higher resolution settings. ${ }^{9,26}$ Therefore, the criteria established in this study (Table 1) could be used when assessing images obtained in vivo.

Prior studies have demonstrated that intracranial plaque burden, lipid core area, and fibrous cap thickness are closely related to plaque vulnerability. ${ }^{9,20,27}$ Teng et $\mathrm{al}^{9}$ found that culprit lesions (ie, lesions in patients with clinical symptoms arising on the side referable to the location of an ischemic stroke on neuroimaging) had greater plaque burden compared with nonculprit lesions in the MCA. In a postmortem study of MCA atherosclerosis, plaques containing $>40 \%$ lipid area were more often associated with cerebral infarct. ${ }^{27}$ We found that our ex vivo high-resolution protocol can reliably quantify lipid core area but overestimated overall plaque burden. This finding may be attributed to tissue shrinkage and distortion during histologic processing, which are difficult to account for in our analysis. ${ }^{24}$ Moreover, the accuracy in characterizing fibrous cap thickness was marginal. This finding may be attributed to the thin fibrous cap seen in most intracranial atherosclerotic plaques, which differs from that in carotid atherosclerotic disease. Therefore, it would be more challenging for in vivo imaging protocols (typically with a resolution of $0.4 \times 0.4 \times 2 \mathrm{~mm}^{3}$ for $2 \mathrm{D}$ imaging $)^{28}$ to visualize a thin fibrous cap.

Calcification area was slightly overestimated by MR imaging; this overestimation was similar to previous findings from an in vivo MR imaging study of carotid plaque. ${ }^{23}$ This is because due to partial volume averaging, voxels that only partially contain calcification can appear hypointense on MR imaging. In general, due to the short $\mathrm{T} 2$ and $\mathrm{T}^{*}$ of calcium, it is challenging for MR imaging to depict calcifications. Also, histology could possibly underestimate the amount of calcification because specimen processing included decalcification before tissue sectioning.

It has been shown that intraplaque hemorrhage is a high-risk feature in the carotid plaque. ${ }^{29}$ However, intraplaque hemorrhage was not present in any of the lesions in this study. The reason could be that plaques obtained in this study were mostly asymptomatic and stable. However, it is also possible that the 
mechanism of stroke caused by intracranial arterial plaque is different from that in carotid plaques because it has been reported that there is a lower incidence of intraplaque hemorrhage in intracranial plaques. ${ }^{8}$

Multicontrast MR imaging has been used for plaque composition characterization due to the limitation of 1 single sequence to distinguish all plaque components. ${ }^{30-33}$ Our results showed that the fibrous cap yielded higher signal intensities than the lipid core on T2-weighted, PD, and STIR sequences, and calcification showed the lowest signal intensity on T2-weighted, PD, and T1-weighted sequences; these findings are similar to previous
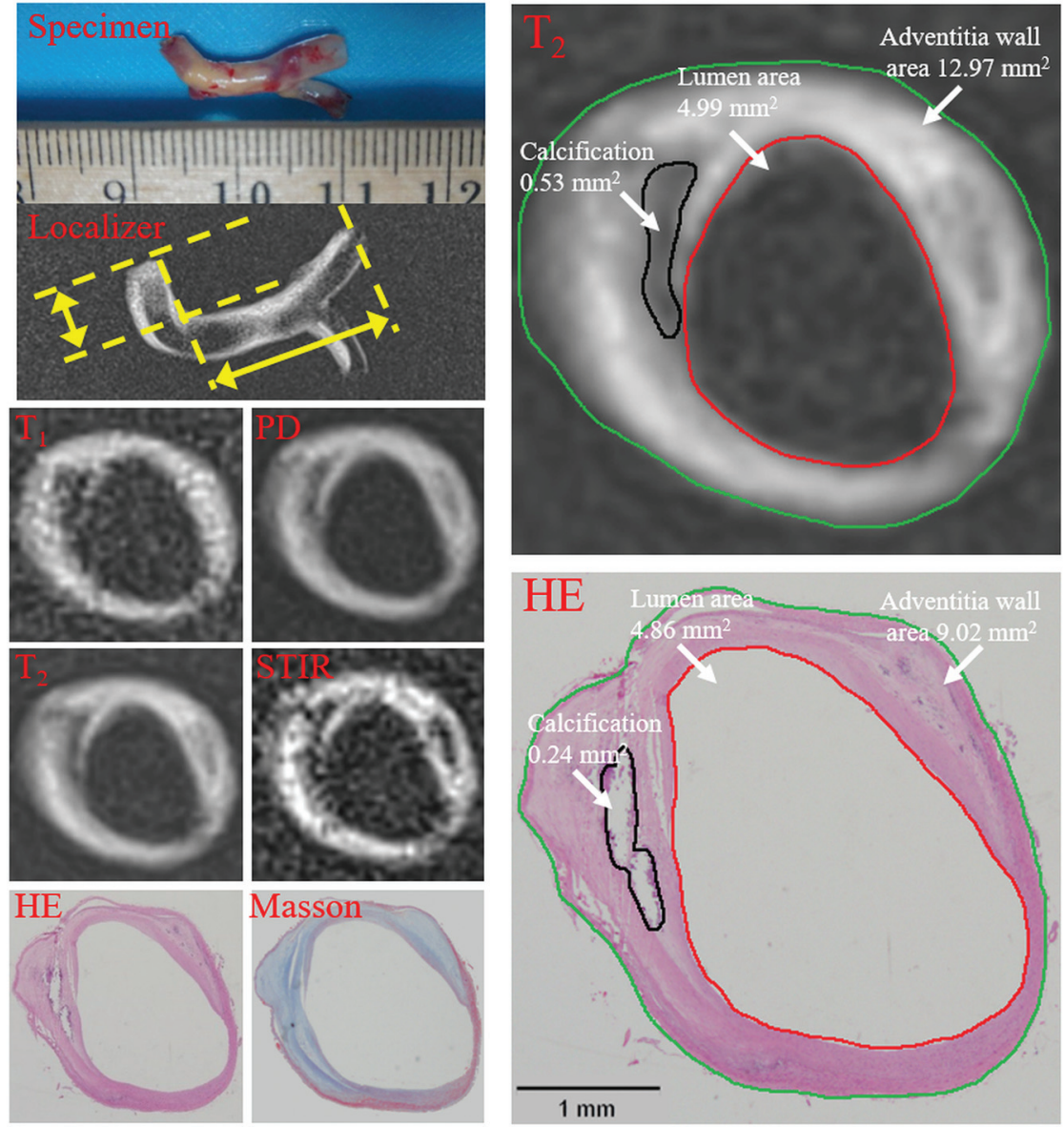

FIG 2. Vertebral artery plaque with fibrous tissue and calcification was identified on multicontrast MR imaging and matched histology (20X magnification). Contours have been drawn for lumen (red), outer vessel wall (green), and calcification (black) on T2-weighted imaging and a hematoxylin-eosin-stained section. The lumen, total vessel, and calcification areas were 4.99, 12.97 , and $0.53 \mathrm{~mm}^{2}$ on MR imaging and $4.86,9.02$, and $0.24 \mathrm{~mm}^{2}$ on histology. The vessel wall areas calculated by total vessel area minus lumen area were 7.98 and $4.16 \mathrm{~mm}^{2}$. The fibrous component area calculated by vessel wall area minus the calcification area was 7.45 and $3.93 \mathrm{~mm}^{2}$. The percentages of components (Specific Component Area / Vessel Wall Area $\times 100 \%$ ) were $6.64 \%$ versus $5.73 \%$ for calcification and $93.36 \%$ versus $94.27 \%$ for fibrous tissue. Plaque burden (Vessel Wall Area / Total Vessel Area $\times 100 \%$ ) was $61.52 \%$ versus $46.09 \%$. observations in carotid imaging. ${ }^{30,31}$ The best contrast and image quality were observed on T2-weighted imaging; thus, the segmentation of the fibrous cap and lipid core was performed on T2-weighted images. Lipid core and fibrous cap were difficult to distinguish on T1-weighted images; this finding agrees with those in previous carotid studies. ${ }^{31,33}$ Although the T1 values of the lipid core and fibrous cap might be different, ${ }^{17}$ the clinical T1-weighted FSE sequence is known to have reduced $\mathrm{T} 1$ contrast because of the long echo train.

Intracranial atherosclerosis is increasingly recognized as an important cause of stroke worldwide. As with carotid atherosclerosis, there is increasing recognition that aggressive (dual) antiplatelet therapy may be warranted and may mitigate the risk of major events such as stroke. However, aggressive medical management is not without risk; therefore, aggressive medical management for intracranial atherosclerosis should be targeted to the patient population in whom the benefit exceeds the risks (eg, bleeding) associated with antiplatelet therapy. Imaging of intracranial atherosclerosis may serve a role in stratifying patients into groups with differing levels of risk based on the nature and extent of atherosclerotic plaque. ${ }^{34}$

There are some limitations to this study. First, the properties of postmortem intracranial arteries might be different from those in arteries in vivo because of changes in the chemical nature and structure of the arterial wall and plaque components related to processing such as dehydration and protein degradation, which could potentially affect the accuracy of results. ${ }^{24}$ Second, the sample size was small $(n=20)$. Specifically, only 57 sections could be used for quantitative assessment, and only 10 sections contained calcification, which may bias the results. Third, MR image thickness (1 $\mathrm{mm}$ ) and histologic section thickness (5 $\mu \mathrm{m})$ did not match, especially for complex plaques in which plaque components may vary over a short distance. Fourth, the various inflammatory components of intracranial atherosclerotic

Table 2: Comparison of the percentages of areas and thicknesses and overall plaque burden between MRI and histology ${ }^{\mathrm{a}}$

\begin{tabular}{lccccc}
\hline & MRI & Histology & $\boldsymbol{P}$ & $\boldsymbol{r}$ & ICC (95\% CI) \\
\hline Fibrous component area (\%) & $81.86 \pm 10.59$ & $81.87 \pm 11.59$ & .997 & 0.901 & $0.898(0.832-0.938)$ \\
Lipid core area (\%) & $19.51 \pm 10.76$ & $19.86 \pm 11.56$ & .660 & 0.888 & $0.885(0.804-0.934)$ \\
Calcification area (\%) & $9.68 \pm 5.21$ & $8.83 \pm 5.67$ & .030 & 0.933 & $0.930(0.745-0.982)$ \\
Fibrous cap thickness (\%) & $31.10 \pm 11.28$ & $30.83 \pm 8.51$ & .890 & 0.438 & $0.421(0.155-0.630)$ \\
Plaque burden area (\%) & $65.18 \pm 9.01$ & $52.71 \pm 14.58$ & $<.001$ & 0.862 & $0.771(0.640-0.858)$ \\
\hline
\end{tabular}

Note:- $r$ indicates the Pearson correlation coefficient.

${ }^{\text {a }}$ Results are expressed as mean \pm SD with $P$ values derived from a linear mixed-effects model. 
plaque were not included in the analysis. Future in vivo studies could evaluate the inflammatory components by including contrast-enhanced 3D T1 black-blood imaging. ${ }^{35}$ Fifth, sequences with lower resolution as typically used in clinical MR imaging were not evaluated in this study, and future investigation is needed. Also, in this study, no intraplaque hemorrhage was found as previously discussed. Another limitation related to the ex vivo nature of this study is the effect of specimen processing and temperature on plaque size and signal properties. Prior work with carotid atherosclerotic plaque observed small but significant increases in plaque size $(4 \%-7 \%)$ and T2 $(8 \mathrm{~ms})$ in imaging samples at room temperature compared with body temperature. ${ }^{24}$ While many recent ex vivo imaging studies were performed at room temperature, ${ }^{15-17,36}$ scanning specimens at room temperature may result in small increases in plaque volume and can nominally prolong the $\mathrm{T} 2$ of plaque tissue. These potential small errors in measurement would be expected to occur equally across specimens.

\section{CONCLUSIONS}

In the evaluation of intracranial atherosclerosis on ex vivo specimens, 3T high-resolution multicontrast MR imaging can accurately identify different plaque components and quantitatively measure the relative volumes of those components, providing a direct reference for in vivo intracranial plaque imaging.

Disclosures: Yuanliang Jiang—RELATED: Grant: Twelfth Five-Year Plan Medical Key Project of the People's Liberation Army, China (BWS12J026); Shanghai Hospital Development Center grant (SHDC12013110); the National Nature Science Foundation of China (31470910).* Wenjia Peng—RELATED: Grant: Twelfth Five-Year Plan Medical Key Project of the People's Liberation Army, China (BWS12J026); Shanghai Hospital Development Center grant (SHDC12013110); the National Nature Science Foundation of China (31470910).* Chengcheng Zhu—RELATED: Grant: National Institutes of Health grants R01NS059944 and R01HL114118. Qi Liu—RELATED: Grant: Twelfth FiveYear Plan Medical Key Project of the People's Liberation Army, China (BWS12J026), the Emerging Frontier Technology Joint Research Program of Shanghai Municipal Hospital (research grant from Shanghai Hospital Development Center), China (SHDC12013110); and the National Nature Science Foundation of China (31470910).* Jianping Lu-RELATED: Grant: Twelfth Five-Year Plan Medical Key Project of the People's Liberation Army, China (BWS12J026); Shanghai Hospital Development Center grant (SHDC12013110); the National Nature Science Foundation of China (31470910)*. *Money paid to the institution.

\section{REFERENCES}

1. Holmstedt CA, Turan TN, Chimowitz MI. Atherosclerotic intracranial arterial stenosis: risk factors, diagnosis, and treatment. Lancet Neurol 2013;12:1106-14 CrossRef Medline

2. Wong KS, Huang YN, Gao S, et al. Intracranial stenosis in Chinese patients with acute stroke. Neurology 1998;50:812-13 CrossRef Medline

3. Kern R, Steinke W, Daffertshofer M, et al. Stroke recurrences in patients with symptomatic vs asymptomatic middle cerebral artery disease. Neurology 2005;65:859-64 CrossRef Medline

4. Bodle JD, Feldmann E, Swartz RH, et al. High-resolution magnetic resonance imaging: an emerging tool for evaluating intracranial arterial disease. Stroke 2013;44:287-92 CrossRef Medline

5. Klein IF, Lavallée PC, Touboul PJ, et al. In vivo middle cerebral artery plaque imaging by high-resolution MRI. Neurology 2006;67: 327-29 CrossRef Medline

6. Swartz RH, Bhuta SS, Farb RI, et al. Intracranial arterial wall imaging using high-resolution 3-Tesla contrast-enhanced MRI. Neurology 2009;72:627-34 CrossRef Medline

7. van der Kolk AG, Zwanenburg JJ, Brundel M, et al. Intracranial vessel wall imaging at 7.0-T MRI. Stroke 2011;42:2478-84 CrossRef Medline
8. Xu WH, Li ML, Gao S, et al. Middle cerebral artery intraplaque hemorrhage: prevalence and clinical relevance. Ann Neurol 2012;71: 195-98 CrossRef Medline

9. Teng Z, Peng W, Zhan Q, et al. An assessment on the incremental value of high-resolution magnetic resonance imaging to identify culprit plaques in atherosclerotic disease of the middle cerebral artery. Eur Radiol 2016;26:2206-14 CrossRef Medline

10. Skarpathiotakis M, Mandell DM, Swartz RH, et al. Intracranial atherosclerotic plaque enhancement in patients with ischemic stroke. AJNR Am J Neuroradiol 2013;34:299-304 CrossRef Medline

11. Vakil P, Vranic J, Hurley MC, et al. T1 gadolinium enhancement of intracranial atherosclerotic plaques associated with symptomatic ischemic presentations. AJNR Am J Neuroradiol 2013;34:2252-58 CrossRef Medline

12. Qiao Y, Zeiler SR, Mirbagheri S, et al. Intracranial plaque enhancement in patients with cerebrovascular events on high-spatial-resolution MR images. Radiology 2014;271:534-42 CrossRef Medline

13. Kim JM, Jung $\mathrm{KH}$, Sohn $\mathrm{CH}$, et al. Intracranial plaque enhancement from high resolution vessel wall magnetic resonance imaging predicts stroke recurrence. Int J Stroke 2016;11:171-79 CrossRef Medline

14. Turan TN, Rumboldt Z, Granholm AC, et al. Intracranial atherosclerosis: correlation between in-vivo $3 \mathrm{~T}$ high resolution MRI and pathology. Atherosclerosis 2014;237:460-63 CrossRef Medline

15. Majidi S, Sein J, Watanabe M, et al. Intracranial-derived atherosclerosis assessment: an in vitro comparison between virtual histology by intravascular ultrasonography, 7T MRI, and histopathologic findings. AJNR Am J Neuroradiol 2013;34:2259-64 CrossRef Medline

16. van der Kolk AG, Zwanenburg JJ, Denswil NP, et al. Imaging the intracranial atherosclerotic vessel wall using 7T MRI: initial comparison with histopathology. AJNR Am J Neuroradiol 2015;36:694701 CrossRef Medline

17. Harteveld AA, Denswil NP, Siero JC, et al. Quantitative intracranial atherosclerotic plaque characterization at $7 \mathrm{~T}$ MRI: an ex vivo study with histologic validation. AJNR Am J Neuroradiol 2016;37:802-10 CrossRef Medline

18. Jiang Y, Zhu C, Peng W, et al. Ex-vivo imaging and plaque type classification of intracranial atherosclerotic plaque using high resolution MRI. Atherosclerosis 2016;249:10-16 CrossRef Medline

19. Naghavi M, Libby P, Falk E, et al. From vulnerable plaque to vulnerable patient: a call for new definitions and risk assessment strategies-Part 1. Circulation 2003;108:1664-72 CrossRef Medline

20. Ravn HB, Falk E. Histopathology of plaque rupture. Cardiol Clin 1999;17:263-70, vii Medline

21. Stary HC, Chandler AB, Dinsmore RE, et al. A definition of advanced types of atherosclerotic lesions and a histological classification of atherosclerosis: a report from the Committee on Vascular Lesions of the Council on Arteriosclerosis, American Heart Association. Arterioscler Thromb Vasc Biol 1995;15:1512-31 CrossRef Medline

22. Cai JM, Hatsukami TS, Ferguson MS, et al. Classification of human carotid atherosclerotic lesions with in vivo multicontrast magnetic resonance imaging. Circulation 2002;106:1368-73 CrossRef Medline

23. Saam T, Ferguson MS, Yarnykh VL, et al. Quantitative evaluation of carotid plaque composition by in vivo MRI. Arterioscler Thromb Vasc Biol 2005;25:234-39 Medline

24. Dalager-Pedersen S, Falk E, Ringgaard S, et al. Effects of temperature and histopathologic preparation on the size and morphology of atherosclerotic carotid arteries as imaged by MRI. J Magn Reson Imaging 1999;10:876-85 CrossRef Medline

25. Gupta A, Baradaran H, Schweitzer AD, et al. Carotid plaque MRI and stroke risk: a systematic review and meta-analysis. Stroke 2013;44: 3071-77 CrossRef Medline

26. Zhang X, Zhu C, Peng W, et al. Scan-rescan reproducibility of high resolution magnetic resonance imaging of atherosclerotic plaque in the middle cerebral artery. PLoS One 2015;10:e0134913 CrossRef Medline 
27. Chen XY, Wong KS, Lam WW, et al. Middle cerebral artery atherosclerosis: histological comparison between plaques associated with and not associated with infarct in a postmortem study. Cerebrovasc Dis 2008;25:74-80 Medline

28. Dieleman N, van der Kolk AG, Zwanenburg JJ, et al. Imaging intracranial vessel wall pathology with magnetic resonance imaging: current prospects and future directions. Circulation 2014;130:192201 CrossRef Medline

29. Saam T, Hetterich H, Hoffmann V, et al. Meta-analysis and systematic review of the predictive value of carotid plaque hemorrhage on cerebrovascular events by magnetic resonance imaging. J Am Coll Cardiol 2013;62:1081-91 CrossRef Medline

30. Clarke SE, Hammond RR, Mitchell JR, et al. Quantitative assessment of carotid plaque composition using multicontrast MRI and registered histology. Magn Reson Med 2003;50:1199-208 CrossRef Medline

31. Fabiano S, Mancino S, Stefanini M, et al. High-resolution multicontrast weighted MR imaging from human carotid endarterectomy specimens to assess carotid plaque components. Eur Radiol 2008;18: 2912-21 CrossRef Medline
32. Shinnar M, Fallon JT, Wehrli S, et al. The diagnostic accuracy of ex vivo MRI for human atherosclerotic plaque characterization. Arterioscler Thromb Vasc Biol 1999;19:2756-61 CrossRef Medline

33. Yuan C, Mitsumori LM, Ferguson MS, et al. In vivo accuracy of multispectral magnetic resonance imaging for identifying lipidrich necrotic cores and intraplaque hemorrhage in advanced human carotid plaques. Circulation 2001;104:2051-56 CrossRef Medline

34. van den Wijngaard IR, Holswilder G, van Walderveen MA, et al. Treatment and imaging of intracranial atherosclerotic stenosis: current perspectives and future directions. Brain Behav 2016;6: e00536 CrossRef Medline

35. Zhu C, Haraldsson $\mathrm{H}$, Tian B, et al. High resolution imaging of the intracranial vessel wall at 3 and $7 \mathrm{~T}$ using 3D fast spin echo MRI. MAGMA 2016;29:559-70 CrossRef Medline

36. Degnan AJ, Young VE, Tang TY, et al. Ex vivo study of carotid endarterectomy specimens: quantitative relaxation times within atherosclerotic plaque tissues. Magn Reson Imaging 2012;30:1017-21 CrossRef Medline 